
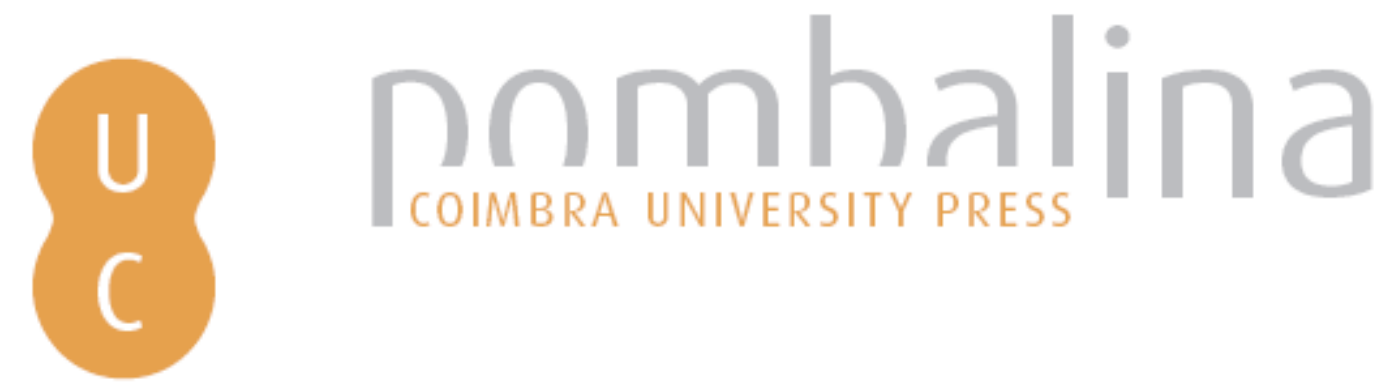

\title{
Enfoques metodológicos y perspectivas de investigación sobre la estructura del factor de impacto de las revistas académicas
}

Autor(es): $\quad$ Campanario, Juan Miguel; Coslado, Maria Angeles; Molina, Antonio

Publicado por: Imprensa da Universidade de Coimbra

URL

persistente: URI:http://hdl.handle.net/10316.2/31881

DOI: $\quad$ DOI:http://dx.doi.org/10.14195/978-989-26-0869-3_2

Accessed : $\quad$ 26-Apr-2023 06:02:56

A navegação consulta e descarregamento dos títulos inseridos nas Bibliotecas Digitais UC Digitalis, UC Pombalina e UC Impactum, pressupõem a aceitação plena e sem reservas dos Termos e Condições de Uso destas Bibliotecas Digitais, disponíveis em https://digitalis.uc.pt/pt-pt/termos.

Conforme exposto nos referidos Termos e Condições de Uso, o descarregamento de títulos de acesso restrito requer uma licença válida de autorização devendo o utilizador aceder ao(s) documento(s) a partir de um endereço de IP da instituição detentora da supramencionada licença.

Ao utilizador é apenas permitido o descarregamento para uso pessoal, pelo que o emprego do(s) título(s) descarregado(s) para outro fim, designadamente comercial, carece de autorização do respetivo autor ou editor da obra.

Na medida em que todas as obras da UC Digitalis se encontram protegidas pelo Código do Direito de Autor e Direitos Conexos e demais legislação aplicável, toda a cópia, parcial ou total, deste documento, nos casos em que é legalmente admitida, deverá conter ou fazer-se acompanhar por este aviso. 
Maria Manuel Borges

Elias Sanz Casado

Coordenação

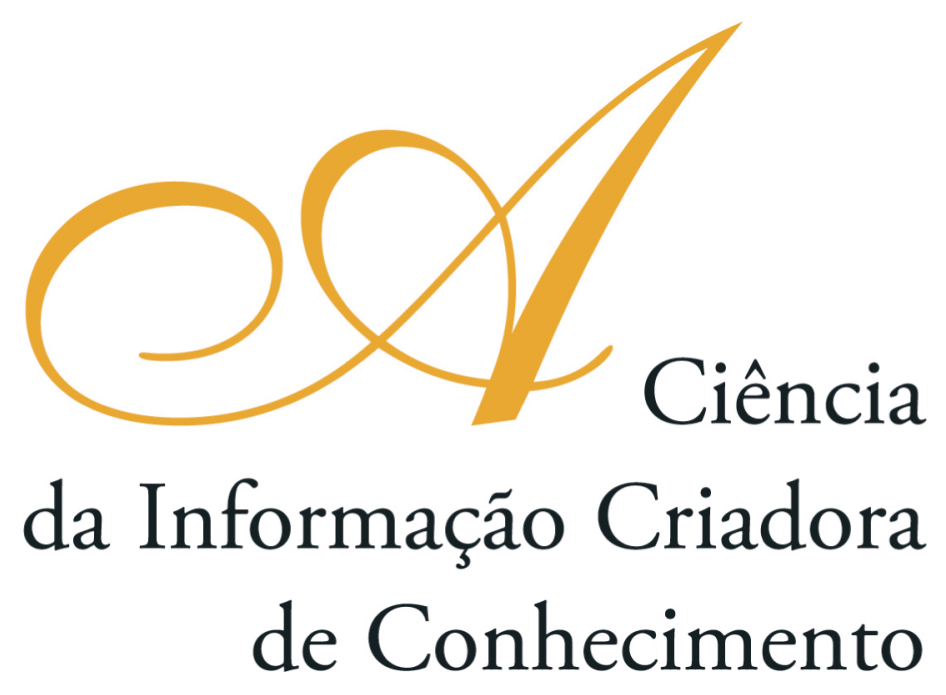

Vol. I I

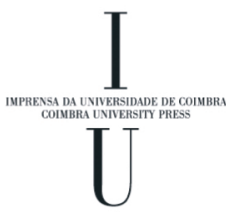

- COImbra 2009 


\section{ENFoques Metodológicos y PERSPECTIVAS de INVESTIgación SOBRe}

LA ESTRUCTURA DEL FACTOR DE IMPACTO DE LAS REVISTAS ACADÉMICAS

Juan Miguel Campanario

Universidad de Alcalá (Espanha)

Maria Angeles Coslado

Fundación Española para la Ciencia y la Tecnología (España)

Antonio Molina

Universidad de Alcalá (Espanha)

\section{Resumen}

En este trabajo se presentan diversas posibilidades de investigación relativas a la estructura del factor de impacto de las revistas académicas. La estructura del factor de impacto se refiere a la descomposición de las citas que contribuyen al factor de impacto de acuerdo con algún criterio que se estime relevante. Se presentan algunos algunos resultados obtenidos hasta ahora por los autores. En general, en los estudios anteriores no se detecta una manipulación masiva del factor de impacto por parte de las revistas científicas.

\section{Abstract}

In this work we introduce some research lines on the structure of impact factors of academic journals. The structure of impact factor refers to the classification of citations that contribute to impact factor according to any relevant criteria. We present some examples of results of research works carried out by the authors. In general, we did not find wide evidence that journals manipulate the impact factors.

\section{Introducción}

El factor de impacto de una revista académica para un determinado año se calcula mediante la siguiente ecuación [Glanzel y Moed, 2002]:

$$
\text { JIF }(\mathrm{Y})=\frac{\text { Citas en } \mathrm{Y} \text { a documentos publicados en } \mathrm{Y} 1 \text { y } \mathrm{Y} 2}{\text { Items citables publicados en } \mathrm{Y} 1 \text { y } \mathrm{Y} 2}
$$

En la ecuación anterior, Y1 e Y2 se refieren a los dos años anteriores a Y. El factor de impacto es uno de los indicadores bibliométricos más utilizado, aunque existe un considerable debate sobre su utilidad y validez. 
Aunque son muchos los artículos que se publican sobre el factor de impacto, no parece que existan muchos trabajos en los que se investigue la estructura de este indicador bibliométrico. De la definición de factor de impacto se desprende que éste consiste esencialmente en un conjunto de citas dividido por un conjunto de ítems citables. La estructura del factor de impacto se refiere a la descomposición de las citas o de los ítems citables siguiendo algún criterio. Por ejemplo, tiene interés estudiar el origen de las citas que contribuyen al factor de impacto de acuerdo con alguna característica o criterio. También puede ser interesante estudiar cómo cambia con el tiempo la estructura de las citas que se utilizan para calcular este indicador.

En la comunicación que se presenta se plantean diversas posibilidades de investigación relativas a la estructura del factor de impacto de las revistas académicas. Estos enfoques metodológicos permiten obtener más información sobre la dinámica de la ciencia y constituyen enfoques de investigación poco utilizados. Las propuestas que se plantean se ilustran con algunos resultados obtenidos hasta ahora por los autores. En particular, se analizan los siguientes enfoques:

a) Análisis de la estructura del factor de impacto en función del tipo de autor de los documentos que aportan citas. Se estudia el efecto de las citas que provienen de artículos publicados por los miembros de los comités editoriales de las revistas académicas cuyo factor de impacto se analiza.

b) Análisis de la estructura del factor de impacto en función de las citas que reciben los artículos publicados en su propia revista por los miembros de los comités editoriales frente a artículos publicados por autores externos a la revista.

c) Efecto de las autocitas en el factor de impacto de revistas en las que este indicador experimenta un crecimiento notable en pocos años.

d) Uso de autocitas de revista en el factor de impacto de revistas en las que este indicador aumenta después de varios descensos sucesivos.

\section{Metodología}

El enfoque general utilizado en todos los casos consiste en clasificar las citas que contribuyen al factor de impacto de acuerdo con algún criterio. Para ello es necesario, en primer lugar, obtener todas las citas que contribuyen al factor de impacto. Este dato aparece publicado en Journal Citation Reports (JCR). En algunos trabajos es necesario conocer la fracción de citas que contribuyen al factor de impacto que son autocitas de revista. En estos casos se recurre a los datos de la matriz "Cited Journal" incluidos en JCR. Para gestionar de forma eficiente estos datos se utilizan funciones y macros del programa Excel. El uso de estas funciones permite obtener datos globales de citas y autocitas de revista.

En algunos de los estudios que se revisan aquí, el análisis se hace en función de la pertenencia al comité editorial de alguno de los autores del documento citante o del documento citado. Para ello es necesario obtener los datos básicos de cada uno de los documentos en cuestión y contrastar los nombres y afiliaciones de cada uno de los autores de estos documentos con los nombres y afiliaciones de los miembros de los comités editoriales de las revistas. Por ejemplo, es necesario comprobar si el autor J Tipler, con afiliación en la Universidad Harvard y que publica un artículo en una 
revista determinada, es el miembro del comité editorial J Tipler, con afiliación en la Universidad Harvard. Esta es una tarea que requiere una gran dedicación e inversión de tiempo, ya que la ambigüedad en algunos casos es considerable. En muchos casos la dificultad puede vencerse gracias a que muchos investigadores suelen incluir su CV investigador en sus páginas web y allí aparecen las publicaciones y se cita la pertenencia a los comités editoriales.

Los estudios que se analizan aquí no requieren la aplicación de cálculos estadísticos sofisticados. En general, basta con obtener tablas de datos globales y porcentajes para cada una de las revistas en cuestión. Estas tablas permiten, por ejemplo, estudiar la variación del porcentaje de autocitas en los años sucesivos. El análisis se completa con gráficas para ilustrar dicha evolución, pero casi nunca se necesita recurrir a pruebas complicadas para contrastar grupos o subgrupos.

\section{Resultados y discusión}

En este apartado se presentan algunos resultados globales de los trabajos realizados en esta línea de investigación.

a) Análisis de la estructura del factor de impacto en función del tipo de autor de los documentos que aportan citas.

En este caso se estudia el efecto de las citas que provienen de artículos publicados por los miembros de los comités editoriales de las revistas académicas. Como es sabido, los miembros de los comités editoriales de las revistas académicas son expertos en sus respectivas áreas de conocimiento y desarrollan tareas de investigación. Estos miembros de los comités editoriales muchas veces publican artículos tanto en la revista donde sirven como en otras revistas. Desde estos artículos pueden citar su propia revista (es decir, la revista en la que figuran como miembros del comité editorial) y estas citas pueden contribuir al factor de impacto [Campanario, González y Rodríguez, 2006]. En este estudio se investigaba el porcentaje de citas que contribuyen al factor de impacto que tienen su origen en documentos en los cuales al menos uno de los autores es miembro del comité editorial de la revista en cuestión.

Para este estudio se seleccionaron 54 revistas de los grupos "Education and Educational Research" y "Psychology, Educational” del Social Sciences Citation Index. Se obtuvo el porcentaje de citas que provenían de artículos en los cuales al menos uno de los autores fuera miembro del comité editorial de la revista en cuestión. El estudio se realizó para los factores de impacto de 2000, 2001 y 2002. El porcentaje de citas que cumplían la condición anterior variaba entre un $0 \%$ y un $61 \%$. Además, en 12 de las revistas analizadas, en al menos uno de los años estudiados, el 50\% o más de las citas que contribuían al factor de impacto provenían de artículos publicados en sus mismas revistas por miembros de su comité editorial. En cuatro de las revistas estudiadas se detectó una cierta acumulación de citas en pocos documentos. Por ejemplo, en sendas revistas se contabilizaron 13 o 16 citas que contribuían al factor de impacto y que provenían de un único documento citante cuyo autor era miembro del comité editorial.

b) Análisis de la estructura del factor de impacto en función de las citas que reciben los artículos publicados por los miembros de los comités editoriales. 
En este caso, el estudio tiene una orientación similar y complementaria al anterior: se trata de averiguar la contribución en citas recibidas por los artículos que publican en su propia revista los miembros de los comités editoriales frente a otros autores. En este caso, las citas que contribuyen al factor de impacto se dividen en dos grupos: citas a artículos cuyos autores son miembros del comité editorial de la revista que se estudia y citas a artículos cuyos autores son externos a la revista [Coslado y Campanario, 2007].

Para este estudio se seleccionaron 44 revistas de los grupos "Education and Educational Research" y "Psychology, Educational” del Social Sciences Citation Index. Sólo se tuvieron en cuenta los documentos etiquetados por Thomson Reuters (antes ISI) como "artículos", ya que éstos son más valorados que otro tipo de publicaciones. Se estudió la contribución de los artículos publicados en 2002 a los factores de impacto de 2003 y 2004. En cada una de las revistas analizadas, los artículos se dividieron en función de sus autores: aquellos que tenían al menos un autor que era miembro del comité editorial frente a artículos en los que todos sus autores eran externos.

En 6 de las revistas analizadas, las citas a artículos escritos por autores externos que contribuyen al factor de impacto son mucho más numerosas que las citas a artículos escritos por miembros del comité editorial. En otras 6 revistas esta pauta se invertía y en el resto de las revistas las contribuciones eran similares.

c) Efecto de las autocitas en el factor de impacto de revistas en las que este indicador experimenta un crecimiento notable en pocos ańos.

Las autocitas a artículos recientes contribuyen al factor de impacto y un abuso de este tipo de citas podría servir para manipular este indicador bibliométrico. Se trata de indagar si las autocitas de revista influyen más que las citas externas en el aumento del factor de impacto en estos casos destacados en que el factor de impacto crece en pocos ańos [Andrade, González-Jonte y Campanario, 2009].

Se estudiaron revistas incluidas en el Science Citation Index en todos los años comprendidos entre 1998 y 2006 y cuyo factor de impacto creció en al menos siete de los ańos siguientes a 1998 y en las que el cociente del factor de impacto de 2006 entre el de 1998 fuese igual o superior a 4. Como puede comprobarse, se trata de revistas en las que el factor de impacto se multiplica en pocos años. Se seleccionaron 43 revistas que cumplían los criterios anteriores. Sin embargo, se estimó conveniente eliminar ocho de las revistas seleccionadas porque el número de citas fue menor de 20 en al menos dos de los años estudiados. Este número tan pequeño podría dar lugar a conclusiones espurias.

Las revistas analizadas fueron clasificadas en diferentes categorías dependiendo de la fracción de autocitas en las citas que contribuyen al factor de impacto. Se encontraron 26 revistas en las cuales la fracción de autocitas fue relativamente pequeña (menos del $30 \%$ en 7 de los 9 años estudiados), mientras que en 4 esta fracción fue elevada (más del 40\% en 7 de los 9 años analizados). El resto de las revistas no encajaba en los patrones anteriores. En 3 de los 4 casos de revistas donde la fracción de autocitas sobre las citas era elevada, el porcentaje de autocitas era superior al 50\% en al menos 8 de los 9 años estudiados. Se trataba de revistas de ámbito preferentemente nacional con un elevado porcentaje de artículos publicados por autores del país donde se editaba.

d) Uso de autocitas de revista en el factor de impacto de revistas en las que este indicador experimenta un crecimiento después de varios ańos de descenso. 
Como es sabido, los investigadores prefieren publicar sus artículos en revistas de alto impacto. Esto es así porque en la evaluación de la actividad investigadora muchas veces las contribuciones se valoran en función del factor de impacto de la revistas donde aparecen. Un descenso continuado de este indicador bibliométrico tendría consecuencias negativas tanto para los autores como para los miembros del comité editorial, ya que la revista perdería prestigio. Si este descenso se prolonga varios ańos, es posible que la revista sea excluida de las bases de datos de Thomson Reuters (antes ISI) y esto supone una auténtica tragedia académica.

En este caso se trata de averiguar si, ante una situación de descenso continuado del factor de impacto, existe algún tipo de «reacción» por parte de las revistas académicas (por ejemplo, un aumento de las autocitas de revista) que lleve a una modificación de la tendencia descendente [Campanario y Molina, 2009]. De nuevo, se trataría de poner en evidencia algún tipo de manipulación del factor de impacto que tuviera como fin corregir una situación de descenso continuado de este indicador.

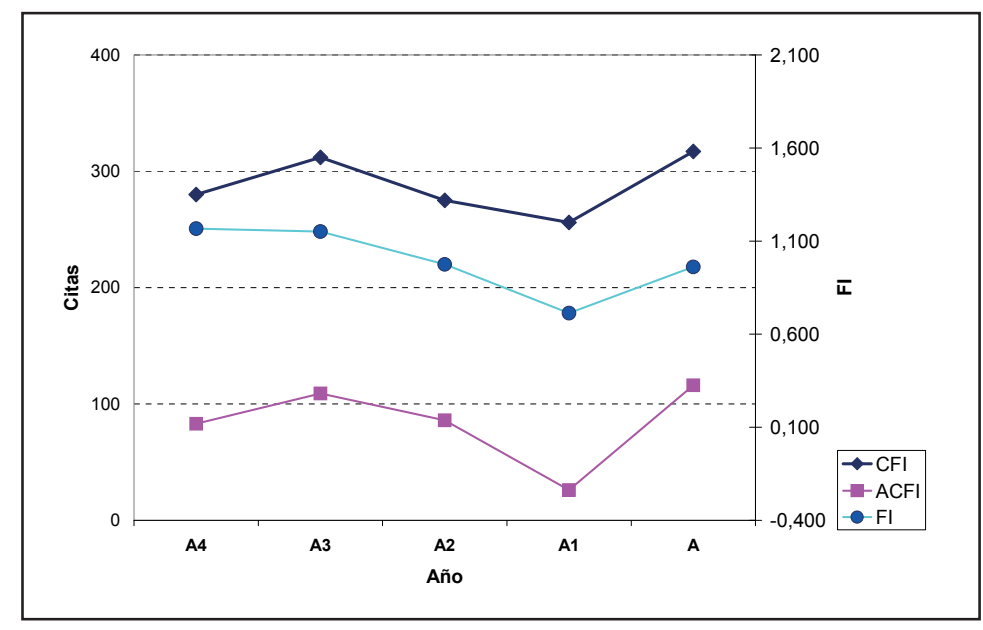

Fig. 1 - Ejemplo de evolución del factor de impacto en una revista determinada (nombre corto Avian Dis) y de algunas variables que se estudian. CFI: Citas que contribuyen al factor de impacto; ACFI: Autocitas que contribuyen al factor de impacto; FI: Factor de impacto.

En este caso se seleccionaron 123 revistas cuyo factor de impacto aumentaba después de cuatro descensos sucesivos. Se eliminaron 16 revistas por las mismas razones que en el estudio anterior. Hay que tener en cuenta que el factor de impacto depende tanto de las citas recibidas (numerador) como de los ítems citables (denominador). El juego de estas dos variables determina los cambios en el indicador bibliométrico. Por ejemplo, el factor de impacto puede aumentar de un ańo para otro porque aumenten las citas, porque disminuyan los ítems citables o por el efecto combinado de ambos cambios. Es posible que un aumento en las citas vaya acompañado de un aumento en los ítems citables y ambos efectos se compensen. Por otra parte, puede haber revistas donde el porcentaje de autocitas sea siempre elevado y no sólo en el año en que se 
produce el cambio de tendencia. Por esta razón, el estudio de los cambios requiere el uso de bastantes variables.

En general, en ese estudio, el aumento del factor de impacto estaba asociado al aumento de las citas. Esto sucedía en un $85 \%$ de las revistas analizadas. Sólo en tres de las revistas se encontró un incremento notable de las autocitas en las citas que contribuyen al factor de impacto justo el año en que se produce el cambio de tendencia. No parece, por tanto, que existan pruebas de una manipulación generalizada de las autocitas para "recuperar" el factor de impacto después de cuatro descensos sucesivos.

\section{Conclusiones}

El factor de impacto es un indicador bibliométrico sobre el que existe una gran controversia [Epstein, 2007, Rossner, Van Epps y Hill, 2007; Sharma, 2007; Walter, Bloch, Hunt y Fisher, 2003; Wilcox, 2008]. Muchos de los comentarios que se publican sobre este indicador son simplemente quejas o descripciones generales, sin que los autores profundicen en su estructura o tengan en cuenta los factores que inciden en los cambios que experimenta.

Los resultados obtenidos hasta ahora en los trabajos revisados ilustran el papel de las estrategias de cita y autocita en revistas académicas, así como algunas peculiaridades propias del proceso de publicación académica en distintas áreas. Para valorar el efecto de las citas que provienen de miembros de los comités editoriales, hay que tener en cuenta que, en revistas de tipo medio, el número de citas que contribuyen al factor de impacto de un año determinado no es muy elevado (del orden de cien o menos). En estos casos, unas cuantas citas que provengan de artículos publicados por miembros de los comités editoriales pueden elevar el factor de impacto de una manera considerable. Esto es así porque este indicador sigue una distribución peculiar, con pocos valores extremos y en la zona media los valores son muy parecidos [Mansilla, Köppen, Cocho \& Miramontes, 2007]. Un aumento en el primer decimal puede colocar a una revista muchas posiciones por delante en el escalafón.

En general, en los estudios anteriores no se detecta un abuso masivo y arbitrario de las autocitas por parte de las revistas científicas. Aunque hay casos de revistas donde la componente de autocitas contribuye considerablemente al factor de impacto, no se trata de una práctica extendida. Hay que tener en cuenta, por otra parte, que un cambio en el factor de impacto depende no sólo del aumento de las autocitas (numerador), sino también del número de ítems citables (denominador). Un editor de una revista interesado en aumentar el factor de impacto puede decidir publicar menos artículos, pero debe estar seguro de que logra seleccionar aquellos que serán citados [Opthof, Coronel \& Janse, 2002].

El análisis de la estructura del factor de impacto constituye un tema relevante de investigación ya que este indicador bibliométrico determina, en gran parte, las estrategias de publicación de muchos científicos y la asignación de posiciones académicas y recursos económicos para la investigación. Conocer de dónde vienen las citas que contribuyen al factor de impacto y cómo evolucionan en diversas circunstancias puede ayudar a valorar mejor este conocido indicador bibliométrico. El estudio de la evolución de la estructura del factor de impacto puede arrojar luz sobre las pautas de comunicación en 
la ciencia y sobre el papel de las políticas editoriales en los procesos de construcción del conocimiento científico.

\section{Referencias bibliográficas}

Andrade, A.; González-Jonte, R.; Campanario, J.M. (2009) Journals that increase their impact factor at least fourfold in a few years: the role of journal self-citations. Scientometrics, 80 , 515-528.

Campanario, J.M.; Molina, A. (2009) Surviving bad times: The role of citations, self-citations and numbers of citable items in recovery of the journal impact factor after at least four years of continuous decreases. Scientometrics, (aceptado para su publicación).

Campanario, JM; González, L; Rodríguez, C (2006) Structure of the Impact Factor of Academic Journals in the Field of Education and Educational Psychology: Citations from Editorial Board Members. Scientometrics, 69, 1, 37-56.

Coslado, MA; Campanario, J.M. (2007) Contribution to the impact factor academic journals in the field of Education and Educational Psychology of citations to articles authored by editorial board members (ISSI-2007, Madrid).

Epstein, D. (2007) Impact factor manipulation. The Write Stuff, 16, 133-134.

Glänzel, W. \& Moed, H. F. (2002) Journal impact measures in bibliometric research. Scientometrics, 53: 171-193.

Mansilla, R.; Köppen, E; Cocho, G.; Miramontes, P. (2007) On the behavior of journal impact factor rank-order distribution. Journal of Informetrics, 1, 155-160.

Opthof, T.; Coronel, R. \& Janse, M.J. (2002) Submissions, impact factor, reviewer's recommendations and geographical bias within the peer review system (1997-2002): Focus on Germany. Cardiovascular Research, 55, 215-219.

Rossner, M., Van Epps, H. \& Hill, E. (2007) Show me the data. Journal of Cell Biology, 179, 1091-1092.

Sharma, O.P. (2007) Journal impact factor: An essential primary quality indicator. Current Science, 93: 5.

Walter, G., Bloch, S., Hunt, G. \& Fisher, K. (2003) Counting on citations: a flawed way to measure quality. Medical Journal of Australia, 178: 280-283.

Wilcox, A.J. (2008). Rise and fall of the Thomson impact factor. Epidemiology, 19, 373-374. 